\title{
Demonstration of the Wide-field Imaging Interferometer Testbed using a Calibrated Hyperspectral Image Projector
}

\author{
Matthew R. Bolcar ${ }^{\mathrm{a}}$, David Leisawitz ${ }^{\mathrm{a}}$, Steve Maher ${ }^{\text {ab }}$, Stephen Rinehart ${ }^{\mathrm{a}}$ \\ aNASA Goddard Space Flight Center, 8800 Greenbelt Rd., Greenbelt, MD 20771 \\ 'Science Systems and Applications, Inc., 10210 Greenbelt Rd., Suite 600, Lanham, MD 20706
}

\begin{abstract}
The Wide-field Imaging Interferometer testbed (WIIT) at NASA's Goddard Space Flight Center uses a dual-Micheison interferometric technique. The WIIT combines stellar interferometry with Fourier-transform interferometry to produce high-resolution spatial-spectral data over a large field-of-view. This combined technique could be employed on future NASA missions such as the Space Infrared Interferometric Telescope (SPIRIT) and the Sub-millimeter Probe of the Evolution of Cosmic Structure (SPECS). While both SPIRIT and SPECS would operate at far-infrared wavelengths, the WIIT demonstrates the dual-interferometry technique at visible wavelengths. The WIIT will produce hyperspectral image data, so a true hyperspectral object is necessary. A calibrated hyperspectral image projector (CHIP) has been constructed to provide such an object. The CHIP uses Digital Light Processing (DLP) technology to produce customized, spectrally-diverse scenes. CHIP scenes will have approximately 1.6-micron spatial resolution and the capability of -producing arbitrary spectra in the band between $380 \mathrm{~nm}$ and 1.6 microns, with approximately 5 -nm spectral resolution. Each pixel in the scene can take on a unique spectrum. Spectral calibration is achieved with an onboard fiber-coupled spectrometer. In this paper we describe the operation of the CHIP. Results from the WIIT observations of CHIP scenes will also be presented.
\end{abstract}

Keywords: Astronomical interferometry, Fourier transform spectroscopy, wide-field imaging, hyperspectral image projectors

\section{INTRODUCTION}

\subsection{The Wide-field Imaging Interferometry Testbed}

The Wide-field Imaging Interferometry Testbed (WIIT) is a facility at NASA's Goddard Space Flight Center, designed to develop and validate a dual-Michelson interferometric technique. By combining Michelson stellar interferometry with Fourier-transform spectroscopy, and using a detector array instead of a single-pixel detector, the WIIT is capable of measuring high-resolution spatial-spectral data over a large field-of-view ${ }^{1}$. Future NASA missions such as the Space Infrared Interferometric Telescope (SPIRIT) and the Sub-millimeter Probe of the Evolution of Cosmic Structures (SPECS) would use this double interferometric technique to observe protostar formation processes and debris disks, developing planetary systems, and distant galaxies in far-infrared and sub-millimeter wavelengths ${ }^{2}$. In the near term, balloon-borne missions such as NASA's Balloon Experimental Twin-Telescope for Infrared Interferometry (BETTII) would use the double interferometric technique to observe clustered star formation ${ }^{3}$.

The goal of the WIIT is to develop the dual-interferometric technique to a Technology Readiness Level (TRL) of 6. By NASA's definition, TRL-6 is achieved when "a high-fidelity system that adequately addresses all critical scaling issues is built and operated in a relevant environment to demonstrate operations under critical environmental conditions"4. The WIIT achieves the "scaling" requirement by operating in the visible wavelength regime and sampling interferometric baselines as long as $230 \mathrm{~mm}$, or at wavelengths approximately $1 / 50^{\text {th }}$ of those to be measured by SPIRIT in the far-infrared with a maximum baseline length of $\sim 36 \mathrm{~m}^{2}$. Critical design parameters such as aperture size, baseline length, and image sampling consequently scale down, allowing the WIIT to fit on a 13' x 5' optical table. The "relevant environment" requirement is achieved by operating in NASA Goddard's Advanced Interferometry and Metrology (AIM) Lab. The AIM lab is a thermally isolated, acoustically stable, Class 10,000 clean room that uses both active and passive

"matthew.bolcar@nasa.gov 
vibration control to provide a quiet environment. Operating in this environment means that all error and noise sources are due to the instrument itself, and can be measured. "Demonstrating operations under critical environmental conditions" is enabled by using a state-of-the-art laser metrology system and encoders to provide adequate feedback for controlling various dynamic processes such as delay-line sweeps and baseline mirror separations ${ }^{5}$.

The only remaining aspect of the TRL-6 definition is the "high-fidelity" requirement. This poses a particular challenge to the WIIT with respect to the types of objects that are used for observation. Since the dual-interferometric technique produces high-resolution spatio-spectrally complex data, it must observe high-resolution spatio-spectrally complex objects representative of the astronomical scenes to be measured by SPIRIT. To achieve TRL-6, the WIIT must be able to observe scenes comprised of numerous objects, both resolved and unresolved, consisting of arbitrarily complex spectra across a large field-of-view. The WIIT achieves this requirement through the use of a Calibrated Hyperspectral Image Projector (CHIP).

\subsection{The Calibrated Hyperspectral Image Projector}

The CHIP is based on an instrument first pioneered by the Optical Technologies Division at the National Institute of Standards and Technology (NIST) ${ }^{6,7,8}$. The basic design uses a combination of Digital Light Processing (DLP) "engines" to produce complex spectra and complex scenes. The hyperspectral image projectors work similarly to commercial video projectors, except the traditional broadband RGB spectral channels are replaced by highly complex, arbitrary spectra.

The NIST group constructed a DLP-based prototype breadboard of a hyperspectral image projector operating in the visible band and are actively pursuing the development of numerous advancements to the technology, including near and mid-infrared capabilities, adaption of the technique to liquid-crystal on silicon (LCOS) technologies, and packaging/design improvements for commercial, industrial, and military applications.

\subsection{Paper Outline}

With guidance from the NIST team, we've built a DLP-based hyperspectral image projector that meets all the requirements of the WIIT project. In Section 2, we provide an overview of WIIT, and Section 3 provides a detailed discussion of the CHIP's operation and its performance. The integration of CHIP with WIIT is discussed in Section 4, including communication and synchronization issues. In Section 5, we show preliminary experimental data and conclude in Section 6.

\section{THE WIDE-FIELD IMAGING INTERFEROMETER TESTBED}

\subsection{WIIT Overview}

Figure 1 shows a diagram of the WIIT optical system. The source plane is located at the focus of a parabolic mirror with a focal length of 2.43 meters. The two, 1-inch interferometer baseline mirrors are mounted on air-bearing stages that allow the baseline separation to be adjusted during data collection. Optical delay is introduced in one arm of the interferometer by means of another air-bearing stage. The beams are combined by a dual-plate beamsplitter and a lens group forms an image of the object at the camera focal plane array.

Control of optomechanical components is provided by a two-tiered metrology system. Absolute positions for the baseline stages and delay stage are provided by an optical encoder system that is accurate to $10 \mathrm{~nm}$. Zygo Distance Measuring Interferometers provide optical path information for each arm of the interferometer, as well as feedback for control of the delay stage?.

The raw datasets produced by the WIIT are 5-dimensional in nature. For a given baseline separation and orientation, images are collected as the delay line is swept through a range of optical path differences relative to the fixed arm. This process is repeated at various baseline separations between $30 \mathrm{~mm}$ and $230 \mathrm{~mm}$, and at various baseline orientations relative to the object (achieved by rotating the object). The field-of-view is sampled with a two-dimensional detector array. The reduction of the 5-dimensional (optical delay, baseline length and angle, and rows and columns of detector pixels) dataset into a 3-dimensional, high-resolution hyperspectral image of the source is discussed in more detail in the companion paper "Wide-field imaging interferometry spatial-spectral image synthesis algorithms" by Richard G. Lyon, et al. ${ }^{10}$. 


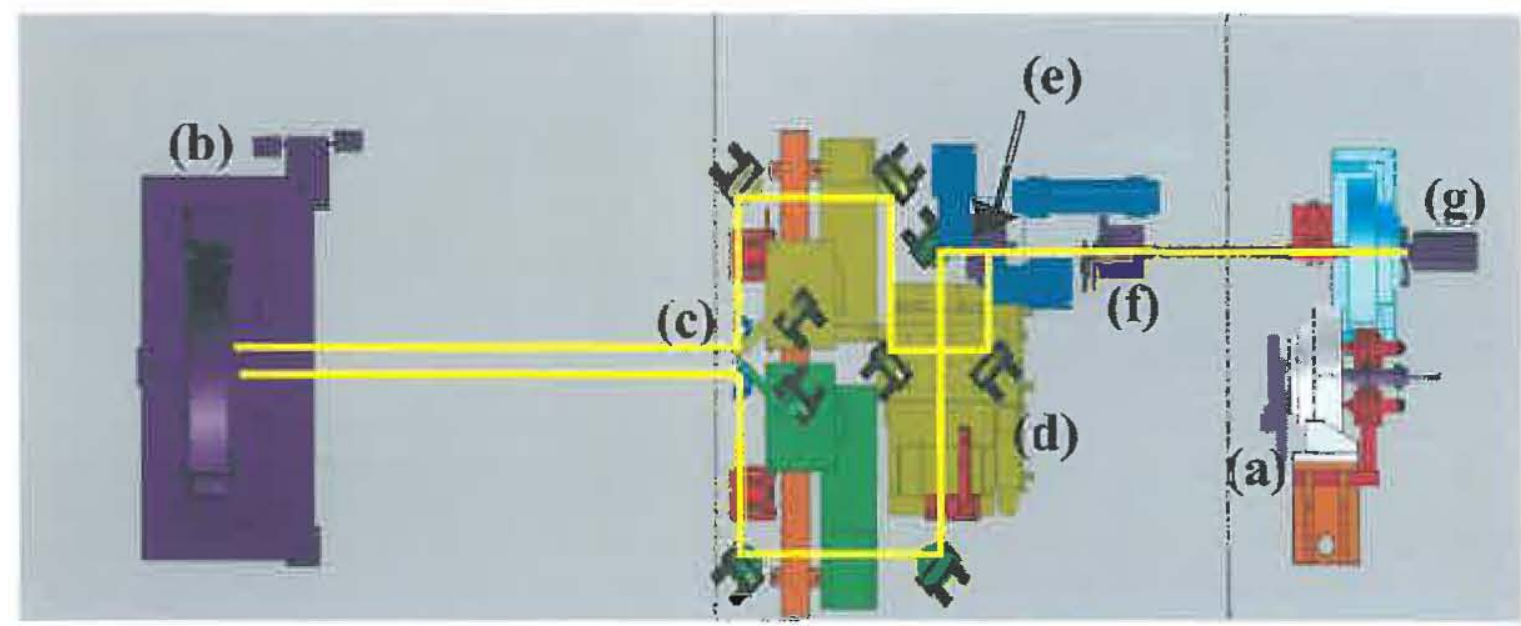

Figure 1-The WIIT optical layout. The source (a) is located at the focus of the collimating mirror (b), which reflects the beam towards the baseline mirrors (c). One arm has a fixed path length, while the other has two fold mirrors on a delay stage (d) to introduce a variable optical path length. The beams are recombined (e) and imaged by a lens group

(f) onto the camera $(\mathrm{g})$. The yellow line illustrates the optical path through the system.

\subsection{WIIT Performance}

The overall performance capabilities of the WIIT are summarized in Table 1. For a more detailed discussion of the performance of the WIIT, see the companion paper "Developing wide-field double-Fourier interferometry for far-IR applications" by David Leisawitz, et al. ${ }^{11}$.

Table 1 - Summary of key WIT performance metrics.

\begin{tabular}{|c|c|}
\hline \multicolumn{2}{|l|}{ Spatial Performance: } \\
\hline Field-of-View (typical): & $2.5 \operatorname{arcmin}$ \\
\hline Plate Scale: & $7.88 \times 10^{-6}$ \\
\hline Pixel size: & $16 \mu \mathrm{m}$ \\
\hline \multicolumn{2}{|l|}{ Resolution (at $400 \mathrm{~nm}$ ): } \\
\hline 30-mm Baseline & $1.38 \operatorname{arcsec}$ \\
\hline 230-mm Baseline & $0.18 \operatorname{arcsec}$ \\
\hline \multicolumn{2}{|l|}{ Spectral Performance: } \\
\hline Operational Range: & $400 \mathrm{~nm}-700 \mathrm{~nm}$ \\
\hline Resolving Power (typical): & $200 @ 550 \mathrm{~nm}$ \\
\hline
\end{tabular}

\section{THE CALIBRATED HYPERSPECTRAL IMAGE PROJECTOR}

\subsection{Theory of Operation}

The CHIP uses two DLP digital micromirror devices (DMD) ${ }^{12}$ to create custom, spectrally diverse, spatially complex scenes. There are two DLP engines, a spectral engine and a spatial engine, that operate in series to produce the scenes. In the spectral engine, a broadband source is dispersed onto the DMD such that individual columns of the mirror are mapped to individual wavelengths. By turning the mirrors in a single column "on", the user includes that specific wavelength in the output. The number of "on" mirrors in a column determines the relative intensity of the spectral component. By selecting numerous columns and appropriately weighting the various spectral components, the user generates a "basis spectrum" of the hyperspectral scene to be projected. 
The spatial engine works very much like a basic video projector. A 2-dimensional binary scene is generated on the DMD by turning the appropriate pixels "on" or "off". Alternatively, an 8-bit gray scale image can be generated using pulse-width modulation to vary the amount of time a particular pixel is "on" relative to the others. Pixels within the DMD array are individually addressed, meaning that arbitrarily complex scenes can be constructed with relative ease.

The spatial and spectral engines are synchronized to produce a hyperspectral scene. Basis spectra are matched with image frames where the intensity of each pixel in the frame determines how much of that basis spectrum is present in the pixel. By cycling through a complete set of frames and their corresponding basis spectra in a period of time that is short compared to the WIIT's integration time, a hyperspectral scene is produced.

\subsection{Design}

CHIP uses two Gooch \& Housego OL490 Agile Light Sources ${ }^{13}$ in the spectral engine. This commercially available solution significantly reduced the development time and cost of the CHIP. One OL 490 operates in the visible regime between $380 \mathrm{~nm}$ and $780 \mathrm{~nm}$, while the other spans the near-infrared (NIR) range between $700 \mathrm{~nm}$ and $1.6 \mu$ m. Even though the WIIT does not operate in the NIR, the capability was included in the CHIP to accommodate future applications at NASA Goddard. Both spectral engines are capable of producing arbitrary spectra with a spectral resolution of approximately $10 \mathrm{~nm}$. The outputs of the two OL490s are combined with a bifurcated fiber bundle, and then coupled to the spatial engine via a liquid light guide (LLG).

The spatial engine of CHIP uses a Texas Instruments DLP Discovery 4100 development kit, which includes a high-speed ALP controller-board that enables the maximum update rate of the DMD ${ }^{14}$. The DMD is an XGA format device, with $1024 \times 768$ pixels that are $10.8 \mu$ m square. With the ALP controller, the full frame of the device can be updated at almost $23 \mathrm{kHz}$, with 1 bit per pixel (i.e. binary frames). Eight-bit gray scale frames can be updated at $291 \mathrm{~Hz}$.

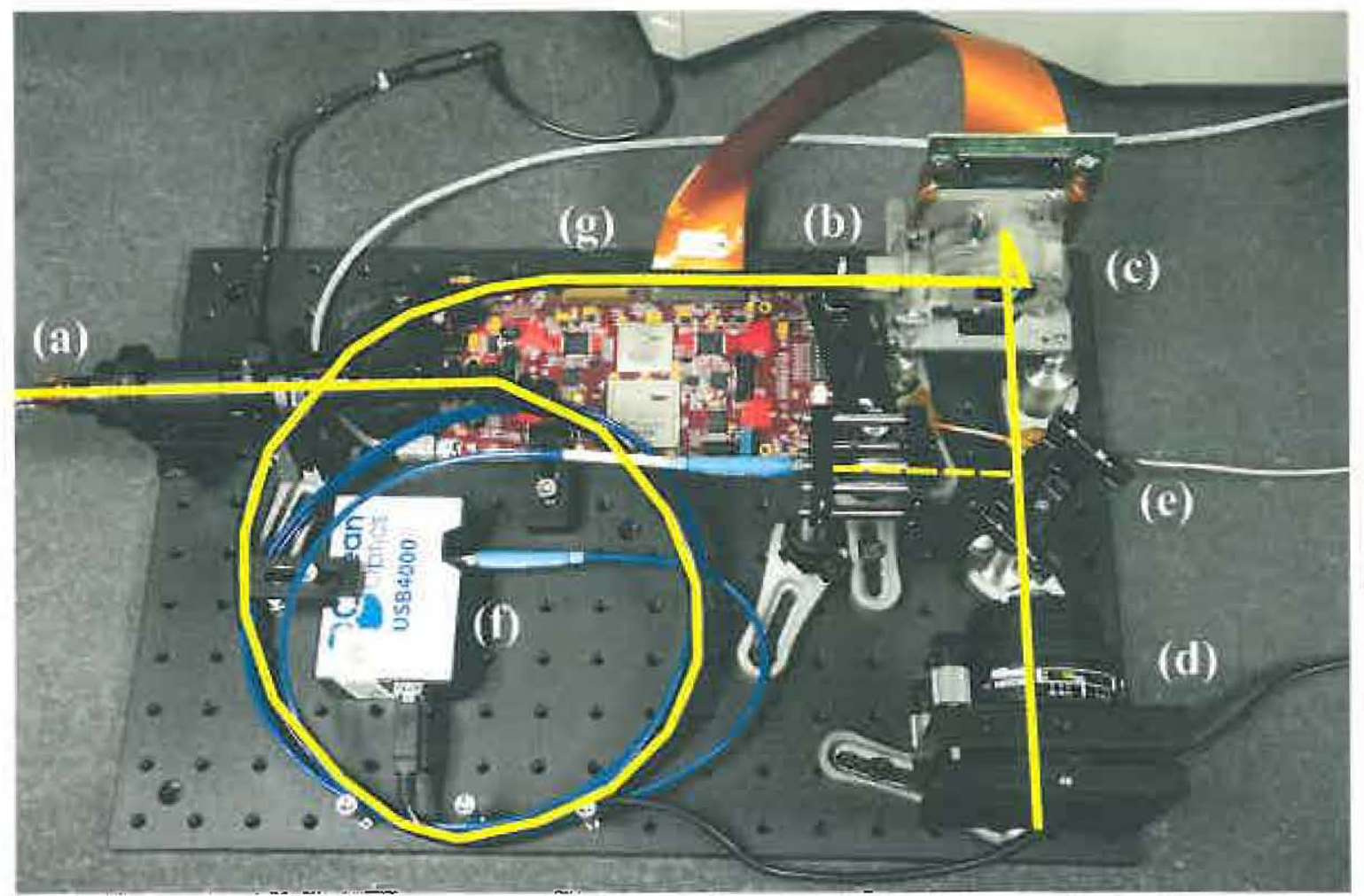

Figure 2 - Photo of CHIP. The OL490 Agile Light Source (not shown) delivers the custom spectral illumination via liquid light guide (a) to the CHIP illumination optics (b). The DLP digital mirror device (c) ${ }^{12}$ is illuminated and imaged by the relay lens (d) ${ }^{15}$ onto the source plane of the WIIT. A flip-in mirror allows the light to be re-directed to an on-board fiber-coupled spectrometer (f) for calibration. The high-speed ALP control board (g) drives the DLP mirror and supplies the master trigger pulse. The yellow line illustrates the optical path through the system. 
Figure 2 shows an annotated photo of CHIP. The output from the OL 490 LLG is coupled into the spatial engine and illuminates the DMD. A Nikkor 28-mm F/2.8 AF-D lens ${ }^{15}$ relays and demagnifies the DMD by a factor of 0.147. The demagnified image has an effective pixel size of $1.6 \mu \mathrm{m}$, and serves as the source scene for the WIIT. An onboard fiber-coupled spectrometer can be used to measure the CHIP illumination and calibrate the spectral output of the OL490s.

Image frames can be pre-generated using a number of methods. Simple shape constructs (i.e. circles, rectangles, etc.) can be generated in arbitrary orientations and arrangements using a JAVA vector graphics engine. Real hyperspectral astronomical data can be reduced using software packages such as ENVI to obtain the required basis spectra and associated abundance frames ${ }^{16}$. All of the image frames and basis spectra are pre-loaded on the ALP controller board and the OL490s, respectively. An external trigger pulse then synchronizes the spatial and spectral engines to ensure the proper spectrum is generated by the OL490s as each frame is projected on the DMD.

Table 2 summarizes the performance characteristics of the CHIP.

Table 2 - Summary of key CHIP performance metrics.

\begin{tabular}{|c|c|}
\hline \multicolumn{2}{|l|}{ Spatial Performance: } \\
\hline Image format: & XGA, $1024 \times 768$ pixels \\
\hline \multicolumn{2}{|l|}{ Pixel size: } \\
\hline Actual: & $10.8 \mu \mathrm{m}$ \\
\hline Effective (after relay lens): & $1.6 \mu \mathrm{m}$ \\
\hline \multicolumn{2}{|l|}{ Frame Rate: } \\
\hline Binary: & $22,727 \mathrm{~Hz}$ \\
\hline 8-bit Gray Scale: & $291 \mathrm{~Hz}$ \\
\hline \multicolumn{2}{|l|}{ Spectral Performance: } \\
\hline Operational Range: & $380 \mathrm{~nm}-1.6 \mu \mathrm{m}$ \\
\hline Update rate: & $12,500 \mathrm{spectra} / \mathrm{s}$ \\
\hline Minimum spectral feature size: & $\sim 10 \mathrm{~nm}$ \\
\hline
\end{tabular}

\section{INTEGRATION WITH THE WIIT}

\subsection{Optical Integration}

As mentioned earlier, a commercial photography lens relays the DMD plane to the WIIT source plane, while demagnifying the DMD by a factor 0.147 . The WIIT collimator and lens system provides an additional demagnification of 0.835 . Therefore, the $10.8-\mu \mathrm{m}$ DMD pixels appear to be $1.3 \mu \mathrm{m}$ at the WIIT camera plane, or about $1 / 12^{\text {th }}$ of a WIIT camera pixel.

As indicated in Table 1, the WIIT plate scale is $7.88 \times 10^{-6}$, or 1.63 arcsec. This is the angle that a single WIIT pixel subtends. A CHIP pixel then appears to subtend $1 / 12^{\text {th }}$ that angle, or 0.135 arcsec. The highest spatial resolution of the WIIT, at a baseline separation of $230 \mathrm{~mm}$ and a wavelength of $400 \mathrm{~nm}$, is 0.18 arcsec. Therefore, a single CHIP pixel remains unresolved by WIIT. This is important in that it allows for calibration of the interferograms collected by the WIIT.

\subsection{Timing Considerations}

The timing associated with the projection of a complete hyperspectral scene and the integration time of the WIIT camera must be carefully controlled. Since a single hyperspectral scene is formed from the projection of individual spectral frames in rapid succession, it is possible to temporally alias the collection of hyperspectral data. To avoid aliasing, the integration time of the WIIT camera must be long enough that each frame of the hyperspectral scene is displayed for an equal amount of time.

For example, assume that a single hyperspectral scene is comprised of 6 basis spectra and their associated 8-bit gray scale frames. The CHIP frame rate in this case is $291 \mathrm{~Hz}$, meaning that complete hyperspectral scenes can be projected at a rate of $291 \mathrm{~Hz} / 6=48.5 \mathrm{~Hz}$. Therefore, in order to assure that a single integration of the WIIT camera "sees" all 6 frames, the camera exposure cannot be shorter than $20.6 \mathrm{~ms}$. If a longer exposure is required to improve 
signal-to-noise ratio, it must be an integer multiple of this minimum value. An exposure of $41.2 \mathrm{~ms}$ would allow each frame in the hyperspectral scene to be displayed exactly twice; an exposure of $82.4 \mathrm{~ms}$ would allow each frame to be displayed three times, and so on.

Figure 3 shows a basic timing diagram illustrating this process. Notice that it is not important that a camera integration begin exactly when a hyperspectral scene begins, only that all of the frames within the scene are captured during a single integration.

To ensure all the frames in the hyperspectral scene are captured correctly, the WIT camera is synchronized with the CHIP using an Arduino Uno microcontroller. The Arduino monitors electrical pulses from the ALP controller, which generates the pulses at the beginning of each frame of the scene. By counting the frame pulses and knowing how many frames comprise a scene, the Arduino knows when a full scene has been projected. The Arduino then provides an external exposure trigger on the WIIT camera, directing the WIIT camera to integrate over a complete hyperspectral scene (again, we can be phase-shifted by any number of frames without harm).

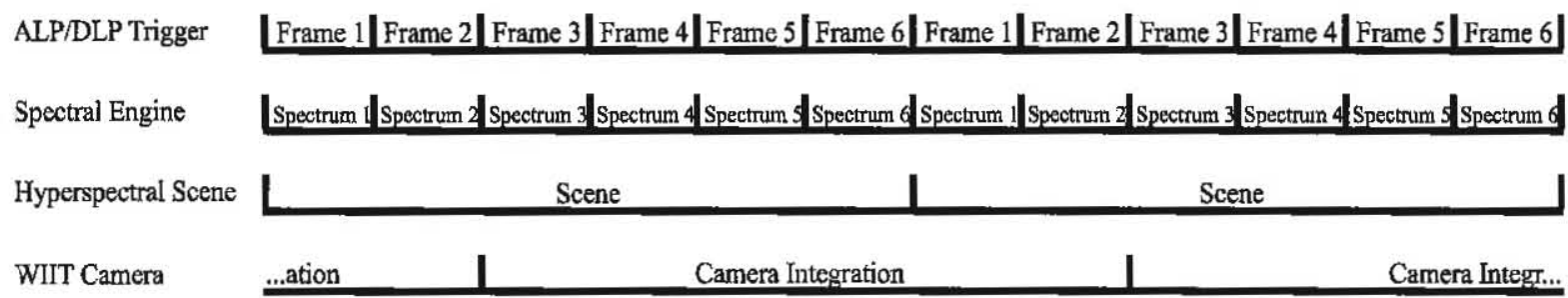

Figure 3-CHIP timing diagram. The ALP/DLP board acts as the "master" clock, generating a trigger pulse at each new frame that advances the spectral engine in sync. A hyperspectral scene consists of several frames (in this case, six). The Arduino microcontroller counts frame triggers and controls the camera integration to insure that a complete scene is captured during the integration. Note that it is not important that a camera integration period begin with the projection of a scene, just that all of the frames are displayed during a single integration.

\section{PRELIMINARY EXPERIMENTAL RESULTS}

Figure 4 shows a photograph of CHIP integrated with the WIIT in the AIM Lab at NASA Goddard Space Flight Center. Figure 5 shows an example scene projected by CHIP and viewed with the WIIT camera. The scene consists of a circle with a radius of $10 \mathrm{CHIP}$ pixels, and a rectangle that is $10 \times 20 \mathrm{CHIP}$ pixels. For this particular scene, both objects had the same spectrum consisting of a $50-\mathrm{nm}$ "top hat" spectrum centered at $\lambda=587 \mathrm{~nm}$.

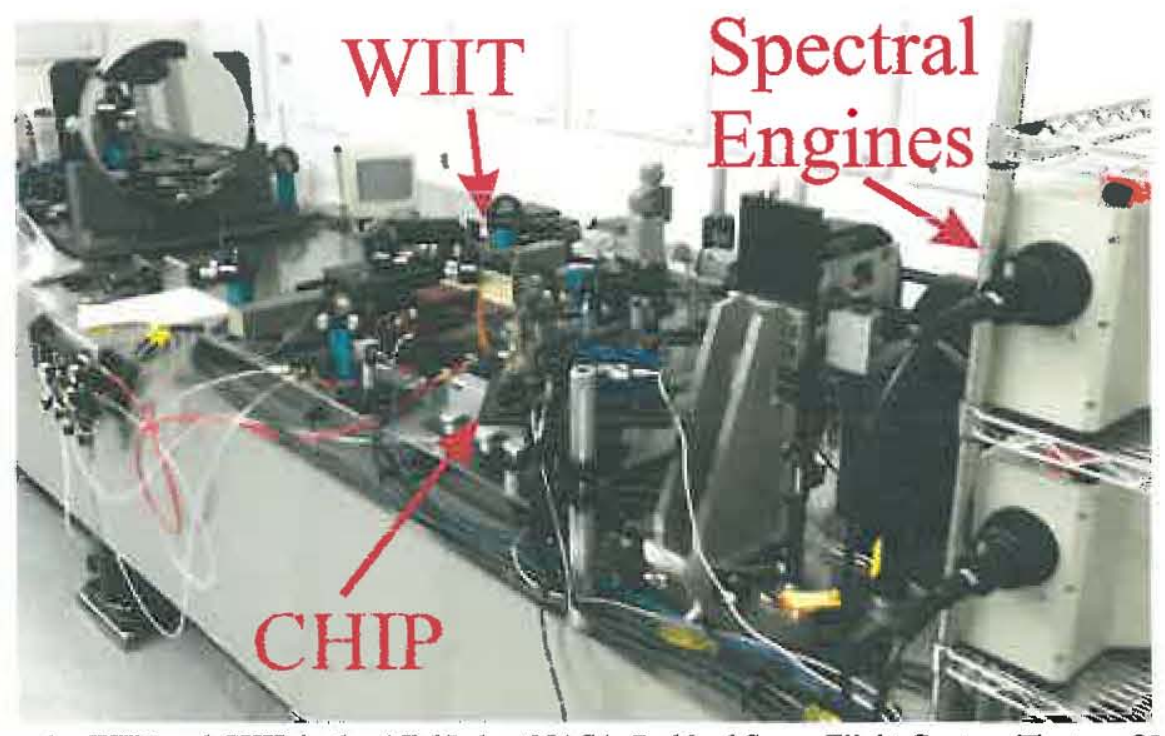

Figure 4 - WIIT and CHIP in the AIM Lab at NASA Goddard Space Flight Center. The two OL490 spectral engines are seen to the right. 


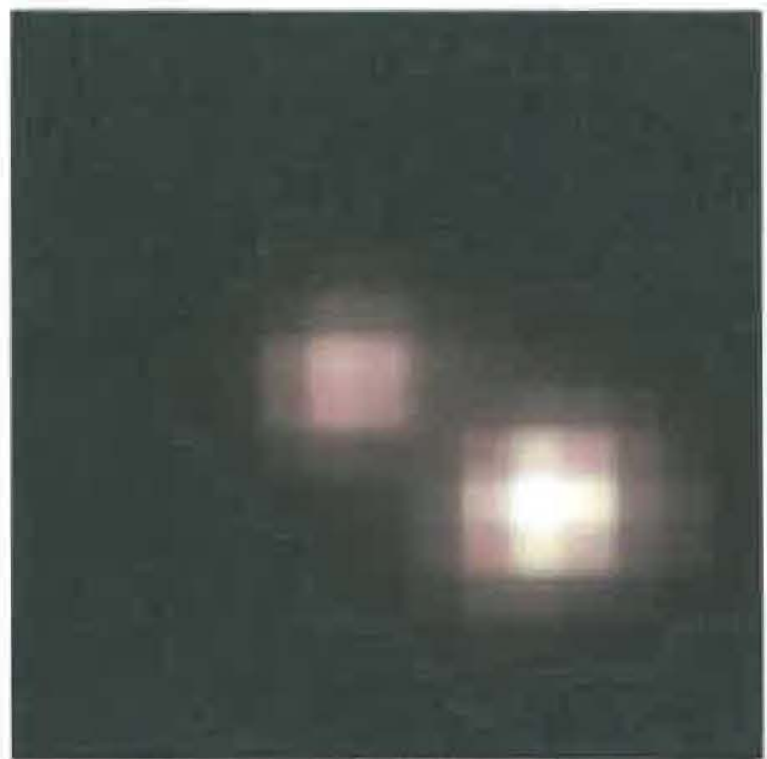

Figure 5-And example CHIP scene as viewed by WIIT. The source to the upper left is a 10-CHIPpixel diameter circle and the source to the lower right is a $10 \times 20$-CHIP-pixel rectangle.

Figure 6 shows the fringe produced as a function of delay-line position for a baseline mirror separation of $30 \mathrm{~mm}$, and a source rotation angle of $0^{\circ}$.

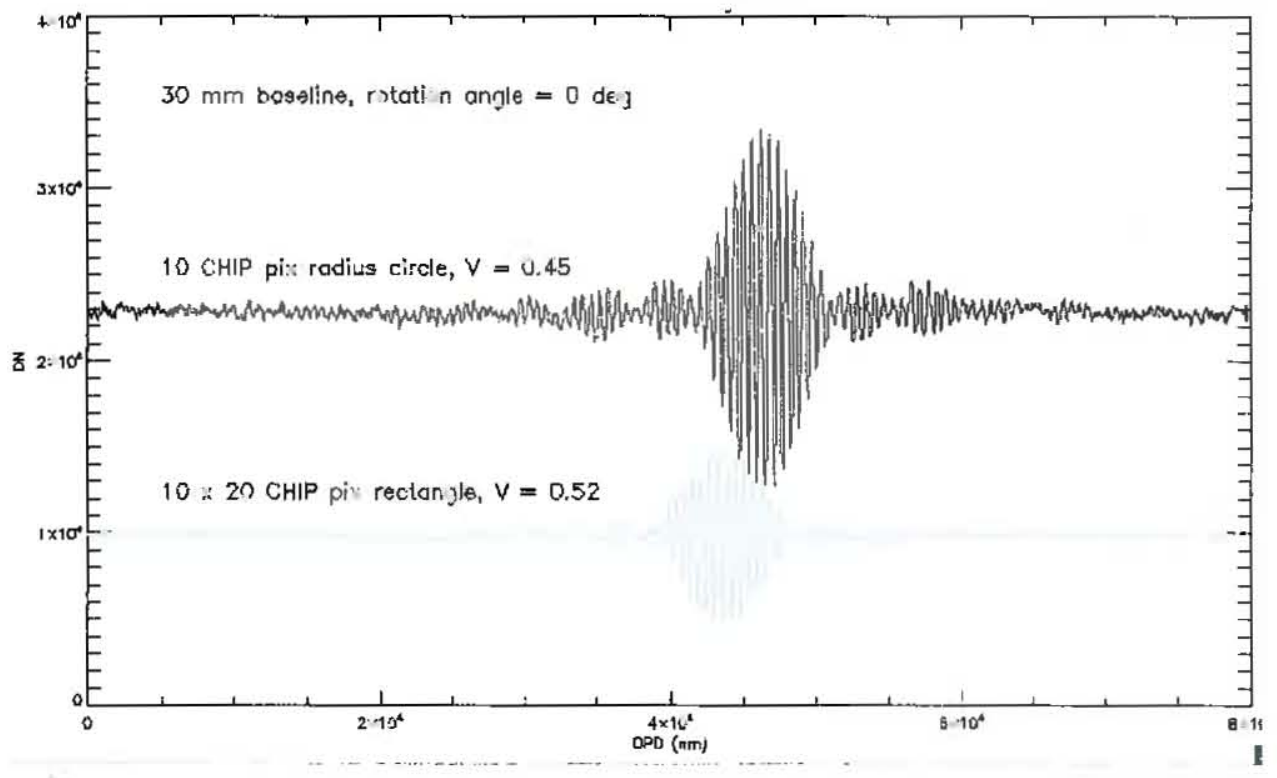

Figure 6 - Example fringes from the CHIP scene.

\section{CONCLUSION}

We described the use of a calibrated hyperspectral image projector to generate realistic, spectrally-diverse, spatiallycomplex scenes as input to the wide-field imaging interferometer testbed. The CHIP uses two DLP-based engines in series to produce the scenes. The spectral engine produces arbitrary spectra in the visible bandwidth between $380 \mathrm{~nm}$ 
and $1.6 \mu \mathrm{m}$, with a spectral resolution of $10 \mathrm{~nm}$. The spatial engine produces 8-bit gray scale $1024 \times 768$ images with an effective pixel size of $1.6 \mu \mathrm{m}$.

The CHIP will be used to develop and validate the double interferometric technique used by the WIIT to produce high-resolution hyperspectral data. The ultimate goal is to achieve a Technology Readiness Level of 6 for the technique, and thereby enable future missions such as SPIRIT and SPECS.

The authors wish to gratefully acknowledge the help of Joe Rice and Steve Brown in the Optical Technologies Division at the National Institute of Standards and Technology for their guidance and advice in the development of the CHIP.

Note: References are made to certain commercially available products to adequately specify the experimental procedures involved. Such identification does not imply recommendation or endorsement by NASA, nor does it imply that these products are the best for the purpose specified.

\section{REFERENCES}

[1] Leisawitz, D., Frey, B., Leviton, D., Martino, A., Maynard, W., Mundy, L, Rinehart, S., Teng, S., and Zhang, X., "Wide-field Imaging Tnterferometry Testbed I: Purpose, Pestbed Design, Data, and Synthesis Algorithms," Proc. SPIE 4852,255 (2003).

[2] Leisawitz, D., et al., "The Space Infrared Interferometric Telescope (SPIRIT): High-resolution imaging and spectroscopy in the far-infrared," Adv. Sp. Res. 40, 689 (2007).

[3] Rinehart, S., et al., "Design and status of the Balloon Experimental Twin Telescope for infrared interferometry (BETTI): an interferometer at the edge of space," Proc. SPIE 8445 (2012).

[4] from NASA Procedural Requirement 7120.8, Appendix J: Technology Readiness Levels (TRLs), 2008, available at http://nodis3.gsfc.nasa.gov/npg_img/N_PR_7120_0008_/N_PR_7120_0008_AppendixJ.pdf.

[5] Leviton, D.B., Frey, B., Leisawitz, D., Martino, A., Maynard, W., Mundy, L, Rinehart, S., Teng, S., and Zhang, X., "Wide-field imaging interferometry testbed 3: metrology subsystem," Proc. SPIE 4852, 827 (2003).

[6] Rice, J.P., Brown, S.W., Johnson, B.C., and Neira, J.E., "Hyperspectral image projectors for radiometric applications," Metrologia 43, S61-S65 (2006).

[7] Brown, S.W., Rice, J.P., Neira, J.E., Bousquet, R., and Johnson, B.C., "Hyperspectral image projector for advanced sensor characterization," Proc. SPIE 6296, 629602-629601 (2006).

[8] Rice, J.P., Brown, S.W., Neira, J.E., and Bousquet, R.R., "A hyperspectral image projector for hyperspectral imagers," Proc. SPIE 6565, C1-C12 (2007).

[9] http://www.zygo.com/?/met/markets/stageposition/zmi/

[10] Lyon, R.G., Leisawitz, D., Rinehart, S., Memarsadeghi, N., Sinukoff, E., "Wide-field imaging interferometry spatial-spectral image synthesis algorithms," Proc. SPIE 8445 (2012).

[11] Leisawitz, D., Lyon, R.G., Maher, S., Memarsadeghi, N., Rinehart, S., Sinukoff, E., "Developing wide-field double-Fourier interferometry for far-IR applications," Proc SPIE 8445 (2012).

[12] http://www.dlp.com/

[13] http://www.goochandhousego.com/products/systems/spectral-imaging-synthesis/ol-490-agile-light-source

[14] http://www.dlinnovations.com/index.php?page=products\&section=d4100 
[15] http://imaging.nikon.com/lineup/lens/singlefocal/wide/af_28mmf_28d/index.htm

[16] Gruninger, J., Ratkowski, A. J., and Hoke, M. L., "The sequential maximum angle convex cone (SMACC) endmember model," Proc SPIE 5425, 1-14 (2004). 


\section{Demonstration of the Wide-field Imaging Interferometer Testbed using a Calibrated Hyperspectral Image Projector}

Matthew R. Bolcar", Lavid Leisawitz", Steve Maher"; Stephen Rinehart"

"NASA Goddard Space Flight Center, 8800 Greenbelt Rd., Greenbelt: MD 20771

Science Systerns and Applications, Inc., 10210 Greenbelt Rd., Sulte 600, Lanham, MD 20706

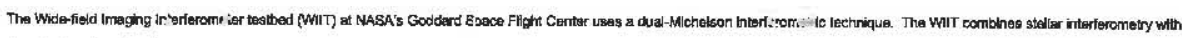

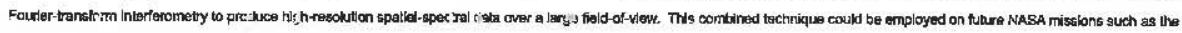

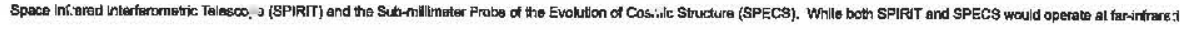

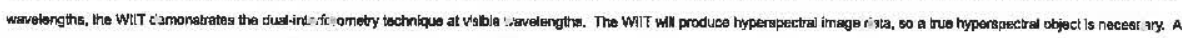

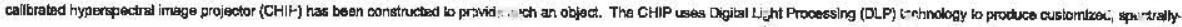

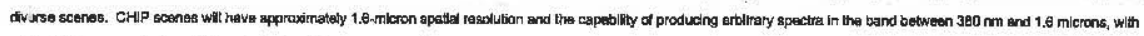

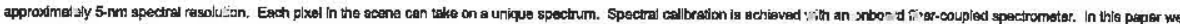
describe the operation of the CHIP. Results from the WIIT absar" thions of $\mathrm{CH}$ HIP scerras will also be presented.

The Wide-ield Imaging Interferometer Testbed

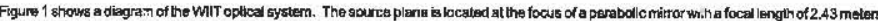

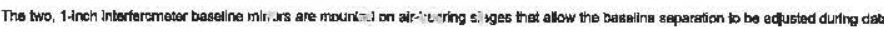

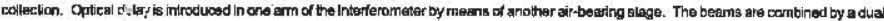

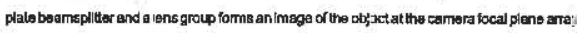

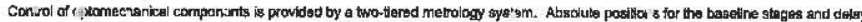

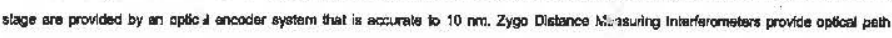

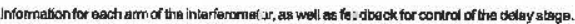

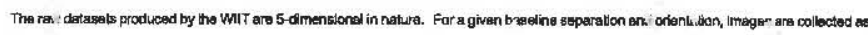

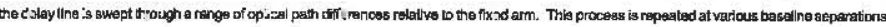

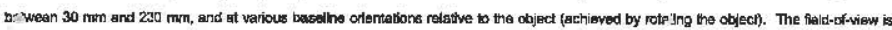

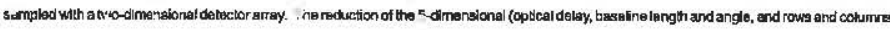

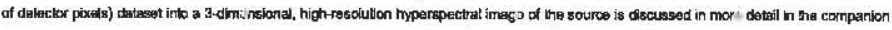

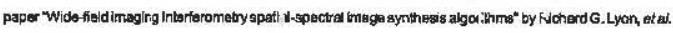

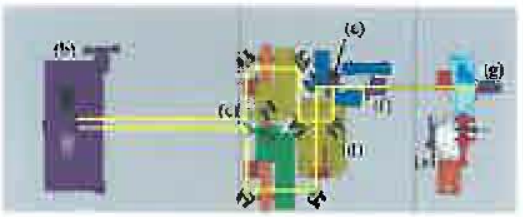

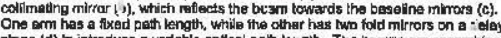

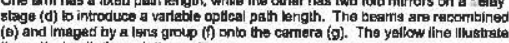

Figure 1 - The MIT aptical layaut. The source (a) is located at the foous of the

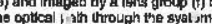

\begin{tabular}{|c|c|}
\hline Spatial Performance & \\
\hline Field-of-Mow \{thpleal\}: & $2.5 \mathrm{mrcmin}$ \\
\hline Pleto Scale: & i.88x $10^{4}$ \\
\hline Pixel Szze: & $16 \mathrm{~mm}$ \\
\hline \multicolumn{2}{|l|}{ Resolution (at $400 \mathrm{~nm}$ ): } \\
\hline 30-mm Baseline & 1.37 ansac \\
\hline 230-mm Bas: îne & 0.58 aresec \\
\hline \multicolumn{2}{|l|}{ Spectral Performance: } \\
\hline Oparational Ranje: & $400 \mathrm{~nm}-700 \mathrm{~nm}$ \\
\hline Rosolwing Powir (thpicalif: & $200500 \mathrm{~nm}$ \\
\hline
\end{tabular}

The Calibrated Hyperspectral Image Projector

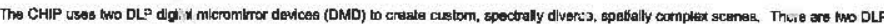

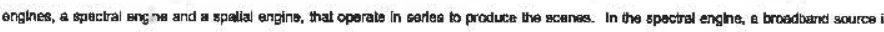

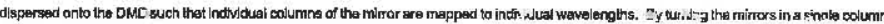

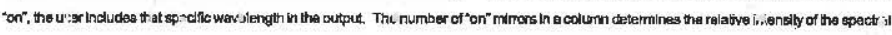

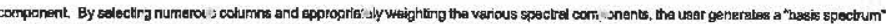

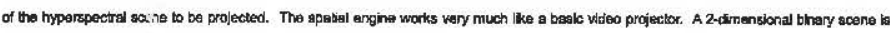

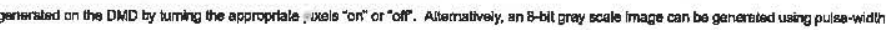

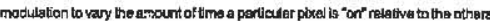

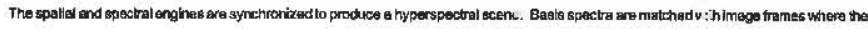

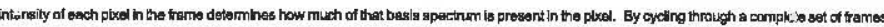

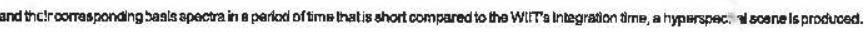

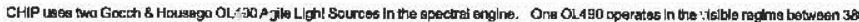

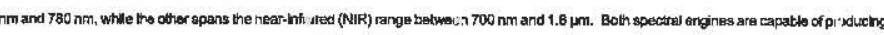

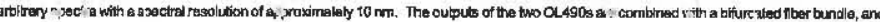
then couplad to the spat:al ongine via a liquid ight gule: (LG).

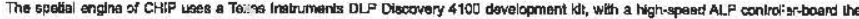

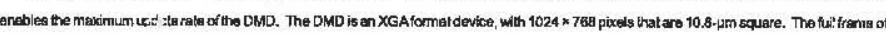

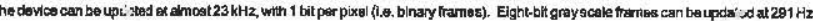

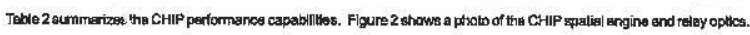

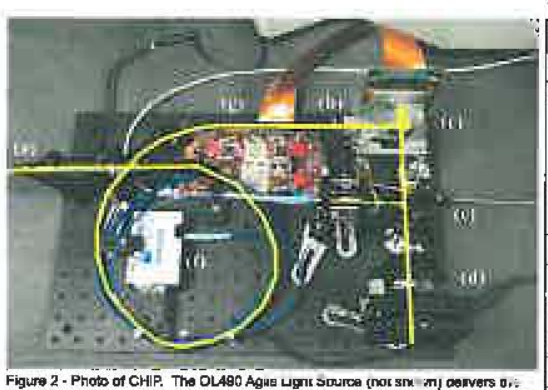
table 2- Summary of CHIP Performance

\begin{tabular}{|c|c|}
\hline \multicolumn{2}{|l|}{ Spattal Perfarmanco } \\
\hline mage Format: & XGA, $1024 \times 768$ F: : : its \\
\hline \multicolumn{2}{|l|}{ Fisel Stze: } \\
\hline Actual: & $10.8 \mu \mathrm{ns}$ \\
\hline Eifective (aftiar relay lens? & $1.6 \mathrm{~mm}$ \\
\hline \multicolumn{2}{|l|}{ Frame Rata: } \\
\hline Enary: & $22,727: i z$ \\
\hline 8-but Gray Scale: & 2019 riz \\
\hline spectral Portormance: & \\
\hline Oporrational Range: & $380 \mathrm{~nm} \cdot 1.6 \mathrm{pm}$ \\
\hline Updato Raie: & 12,500 spactra/s \\
\hline Min. Spactral Feature Ske: & $-10 \mathrm{~nm}$ \\
\hline
\end{tabular}

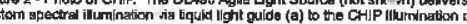

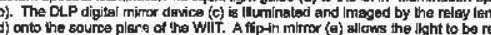
irectad is an an-board fiber-couplad spectrometer (f) for cellonation. The high-

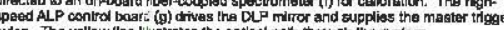

Integration of CHIP with WIIT

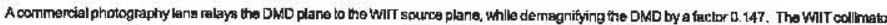

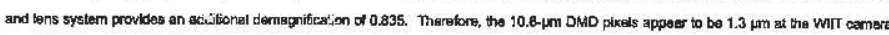

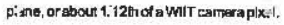

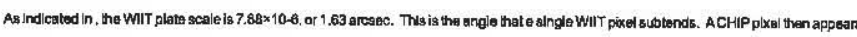

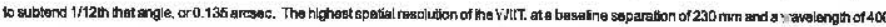

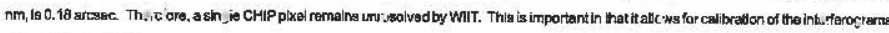
collectod by the wir.

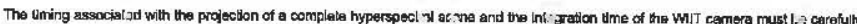

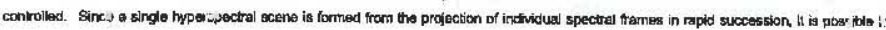

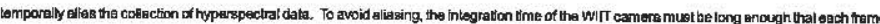
of hine hyperspectirs scens is displayed for anequal amounl of time.

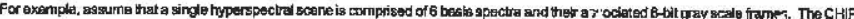

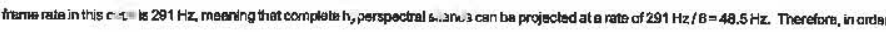

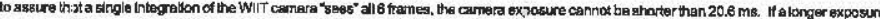

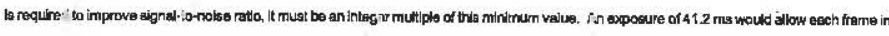

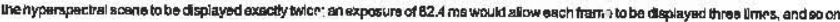

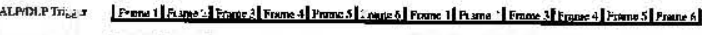

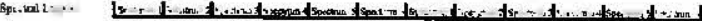

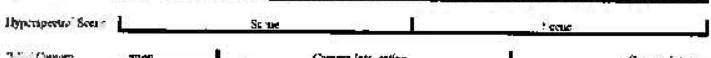

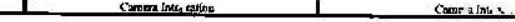

I igure 3 - C. IIP tumlng diagram. The ALPROLP bo:ind acli as the "master" clock, genc aling a trigger pulse at

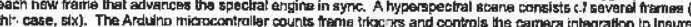

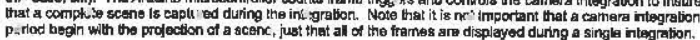

Results

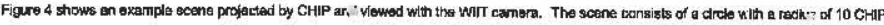

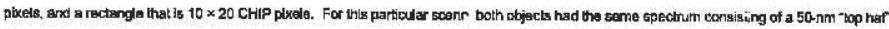

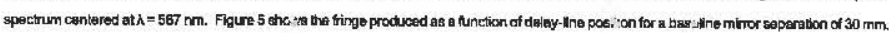
ancte souncerotation engle of $0^{\circ}$

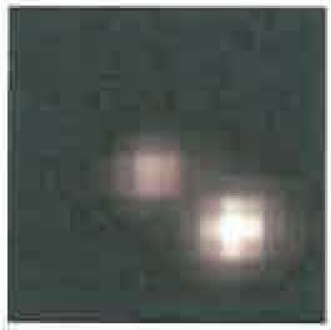

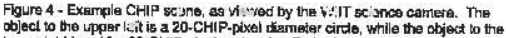
lawer right is a $10 \times 20$ C. HIPP-pixel rectend

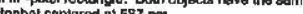

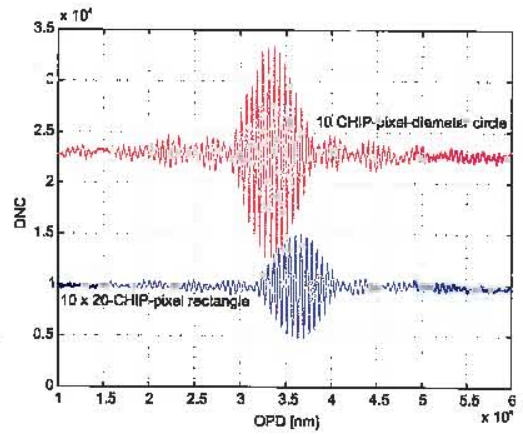

Reference

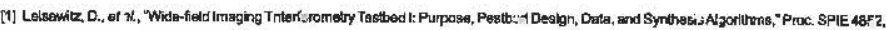
256 (2003)

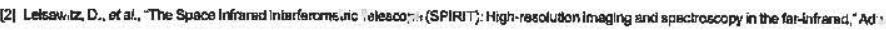
Sp. Ras. 40, 6as (2007).

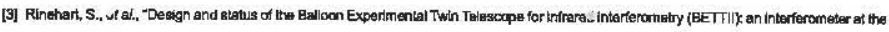
edge of space," Proc. SPE. 8445 (2012)

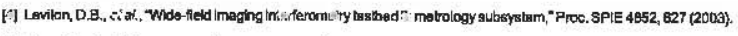

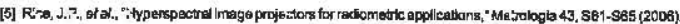

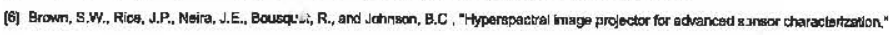
Proc, S.ग. 지 6296, 629602-629601 (2006).

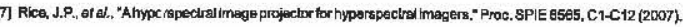

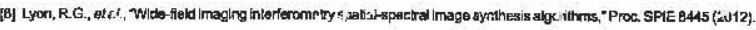

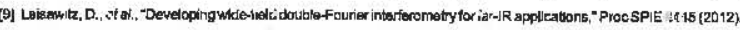

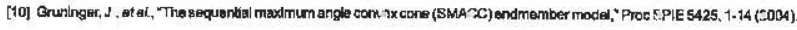

